\title{
THE RADIAL METALLICITY GRADIENTS IN THE MILKY WAY THICK DISK AS FOSSIL SIGNATURES OF A PRIMORDIAL CHEMICAL DISTRIBUTION
}

\author{
A. Curir ${ }^{1}$, A. L. Serra ${ }^{1,2,3}$, A. Spagna ${ }^{1}$, M. G. Lattanzi ${ }^{1}$, P. Re Fiorentin ${ }^{1}$, and A. Diaferio ${ }^{2,3}$ \\ ${ }^{1}$ INAF-Osservatorio Astrofisico di Torino, Italy; curir@oato.inaf.it \\ ${ }^{2}$ Dipartimento di Fisica, Università di Torino, Torino, Italy \\ ${ }^{3}$ Istituto Nazionale di Fisica Nucleare (INFN), Sezione di Torino, Torino, Italy \\ Received 2013 December 6; accepted 2014 February 17; published 2014 March 14
}

\begin{abstract}
In this Letter we examine the evolution of the radial metallicity gradient induced by secular processes, in the disk of an $N$-body Milky Way-like galaxy. We assign a $[\mathrm{Fe} / \mathrm{H}]$ value to each particle of the simulation according to an initial, cosmologically motivated, radial chemical distribution and let the disk dynamically evolve for $\sim 6$ Gyr. This direct approach allows us to take into account only the effects of dynamical evolution and to gauge how and to what extent they affect the initial chemical conditions. The initial $[\mathrm{Fe} / \mathrm{H}]$ distribution increases with $R$ in the inner disk up to $R \approx 10 \mathrm{kpc}$ and decreases for larger $R$. We find that the initial chemical profile does not undergo major transformations after $\sim 6 \mathrm{Gyr}$ of dynamical evolution. The final radial chemical gradients predicted by the model in the solar neighborhood are positive and of the same order as those recently observed in the Milky Way thick disk. We conclude that (1) the spatial chemical imprint at the time of disk formation is not washed out by secular dynamical processes and (2) the observed radial gradient may be the dynamical relic of a thick disk originated from a stellar population showing a positive chemical radial gradient in the inner regions.
\end{abstract}

Key words: Galaxy: abundances - Galaxy: disk - Galaxy: evolution - methods: numerical - solar neighborhood

Online-only material: color figures

\section{INTRODUCTION}

Observations suggest that the negative radial metallicity gradient, $d[\mathrm{Fe} / \mathrm{H}] / d R$, measured in the solar neighborhood close to the plane, flattens or even attains positive values if we select stars at higher $|z|$ from the galactic plane or with high $[\alpha / \mathrm{Fe}]$ abundances (Allende Prieto et al. 2006; Boeche \& the RAVE Collaboration 2013; Anders et al. 2013; Hayden et al. 2013). This effect can be a signature of a positive radial gradient in the thick disk population, as derived by Marsakov \& Borkova (2005) and by Carrell et al. (2012), who analyzed kinematically selected thick disk samples. Alternatively, a similar feature can be simply produced by the variation of the fraction of thin disk and thick disk stars due to their different scale-height and scalelength (Anders et al. 2013). This scenario is indeed consistent with the short scale length, $h_{R} \sim 2 \mathrm{kpc}$, recently estimated for $\alpha$-enhanced stars by Bensby et al. (2011) and Cheng et al. (2012).

Furthermore, there is observational evidence indicating that the Milky Way (MW) is a barred galaxy. Non-axisymmetric structures in the disk (such as bars and spiral arms) drive galaxy evolution: they redistribute angular momentum among the different galactic components and can enhance radial migration inside the disk. The amount of impact that these secular processes can have on the formation of the thick disk and on its chemical and kinematical properties is still a matter of debate (Roškar et al. 2008; Loebman et al. 2011; Brunetti et al. 2011; Minchev et al. 2012; Kubryk et al. 2013).

This work is aimed at analyzing whether dynamical effects can be responsible for the non-negative radial metallicity gradients observed today in the MW thick disk, and suggests that such gradients can be regarded as a cosmological feature.

We use an $\mathrm{N}$-body disk that develops a bar and whose final population displays a radial metallicity gradient; this is obtained from an initial axisymmetric disk with a positive radial metallicity gradient for $R<10 \mathrm{kpc}$, which has dynamically evolved for 6.1 Gyr. This secular evolution model was recently proven (Curir et al. 2012) to reproduce the observed velocitymetallicity correlation in the thick disk (Spagna et al. 2010; Lee et al. 2011). In this Letter, we show that the positive slope of the chemical radial gradient in the MW thick disk observed today in the solar neighborhood might be a signature remaining from the primordial population of disk stars.

\section{NUMERICAL SIMULATIONS AND CHEMICAL TAGGING}

The simulation used in this work was presented in Curir et al. (2012); it is an exponential disk with structural parameters appropriate for an MW-like dark matter (DM) halo at redshift $z=0$. The disk, having a mass of $5.6 \times 10^{10} M_{\odot}$ and scale length $h=3.5 \mathrm{kpc}$, is embedded in a Navarro-Frenk-White DM halo (Navarro et al. 1997) with $10^{7}$ particles having mass $M_{\mathrm{vir}}=10^{12} M_{\odot}$ and concentration $C_{\mathrm{vir}}=7.4$ (see Curir et al. 2012 for details). We ran the simulation using the public parallel treecode GADGET2 (Springel 2005) on the cluster matrix at the CASPUR (Consorzio Interuniversitario per le Applicazioni del Supercalcolo) consortium, Rome. Our simulation can be considered as an evolving thick disk model, consistent with the scenario where the thick disk is originated from gas accreted in a chaotic period at high redshift and with short star formation timescales (Brook et al. 2004, 2012). The initial radial metallicity distribution of the disk can be seen as the link between our isolated simulated disk and the cosmological scenario, as we explain in the following paragraphs.

In order to avoid the effect of relaxation of the initial conditions, we let the dynamical system evolve for $1 \mathrm{Gyr}$. Then the time is set to zero and each particle in the disk is tagged with a $[\mathrm{Fe} / \mathrm{H}]$ label, according to the initial radial function shown in Figure 1. This function is based on Model S2IT from Spitoni 


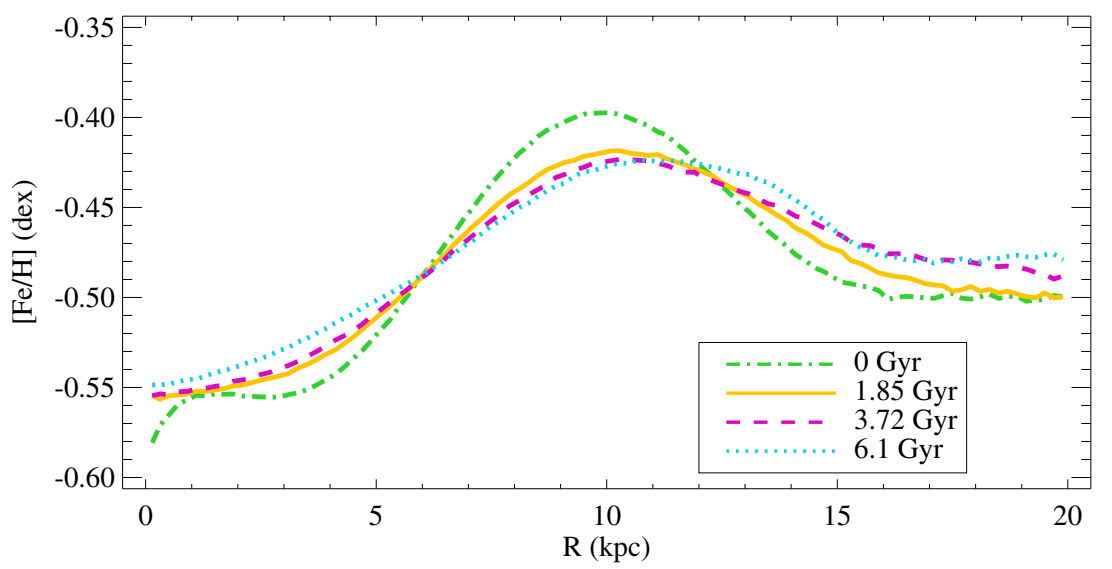

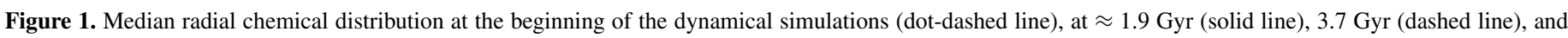
6.1 Gyr (dotted line). The points are the median values of the data over bins $0.2 \mathrm{kpc}$ wide.

(A color version of this figure is available in the online journal.)

\& Matteucci (2011). We assign metallicities with a dispersion $\sigma_{[\mathrm{Fe} / \mathrm{H}]}=0.1 \mathrm{dex}$, which represents the variation of the median chemical profile over $1 \mathrm{Gyr}$, according to Spitoni \& Matteucci (2011). Regarding the metallicity distribution as a function of the height, $z$, from the galactic plane, no initial vertical gradient has been included in our simulation.

The positive slope of the function for $R<10 \mathrm{kpc}$ is justified with an inside-out disk formation model: at early epochs the efficiency of chemical enrichment in the inner regions of the disk is low due to a large amount of infalling primordial gas. This scenario is consistent with recent results published by Cresci et al. (2010), who found evidence for an "inverse" (i.e., positive) metallicity gradient in a sample of galaxies with stellar mass $>3 \times 10^{9} M_{\odot}$, at $z \sim 3$. They conclude that this is produced by the accretion of primordial gas (Mott et al. 2013), which diluted the abundance of elements heavier than helium in the center of the galaxies.

The simulation produces a bar at around 2 Gyr which persists throughout. The radial migration, illustrated in Figure 2, redistributes the angular momenta of the orbits inside and outside the bar. In Figure 2 we can see to what extent the radius of each particle in the simulation has changed from $R_{i}$, at the start of the dynamical time, to $R_{f}: 1.9,3.7$ or $6.1 \mathrm{Gyr}$ later. The stars with $R_{f} / R_{i}<1$ have moved toward inner regions, whereas the other stars have migrated further away. Thus, while the particles in the inner parts have a tendency to go toward the center, the outer regions of the disk are expanding. As for the distribution perpendicular to the plane of the disk, we observe a similar behavior: the internal regions tend to compact with time along $|z|$, whereas, in the external regions the disk particles increase their median $\left|z_{f} / z_{i}\right|$ (e.g., Loebman et al. 2011; Roškar et al. 2013). The profile $\left|z_{f} / z_{i}\right|$ at $6 \mathrm{Gyr}$ is the result of the formation of a boxy bulge, probably due to buckling instabilities, although further analysis is needed in order to provide a full consistent explanation.

\section{RESULTS AND DISCUSSION}

Figure 1 shows the median radial chemical distribution at four different dynamical epochs: $t=0,1.9,3.7$ and 6.1 Gyr. The radial migration flattens out the initial chemical profile in the disk outer region, while it does not induce major modifications in the inner regions. The peak in the metallicity distribution, initially at $9.9 \mathrm{kpc}$, shifts to $10.7 \mathrm{kpc}$ after $\sim 6 \mathrm{Gyr}$; also
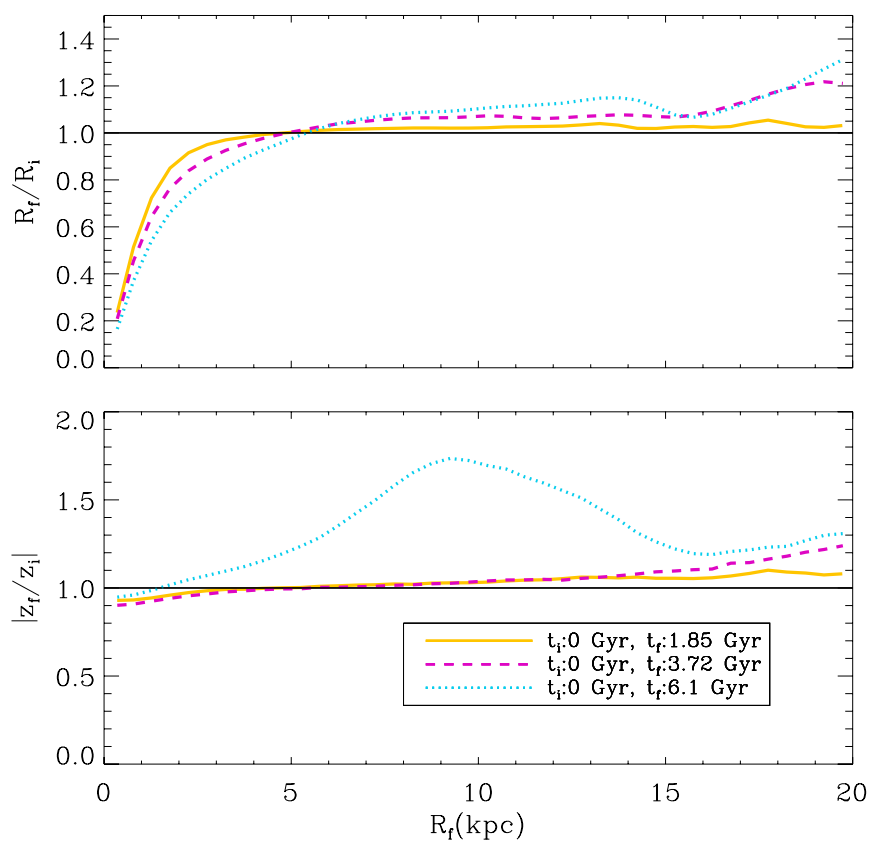

Figure 2. Migration of particles. Top panel: profiles of the median ratio $R_{\mathrm{f}} / R_{\mathrm{i}}$, where $R_{\mathrm{i}}$ is the radius of a particle at initial time and $R_{\mathrm{f}}$ is that of the same particle at 1.85 Gyr (solid line), 3.7 Gyr (dashed line) or 6.1 Gyr (dotted line). Bottom panel: profiles of the median ratio $\left|z_{\mathrm{f}} / z_{\mathrm{i}}\right|$, at the same times. The binning range over the $x$-axis is $0.5 \mathrm{kpc}$ in both panels.

(A color version of this figure is available in the online journal.)

the value of the $[\mathrm{Fe} / \mathrm{H}]$ peak varies from an initial value of $-0.40 \mathrm{dex} \mathrm{kpc}^{-1}$ to $-0.42 \mathrm{dex} \mathrm{kpc}^{-1}$. These relatively small changes point out the fact that dynamics alone can only marginally affect the initial radial chemical distribution.

Figure 3 shows the radial chemical distribution of the simulated disk particles after 6.1 Gyr of dynamical evolution. The particles are taken within a few kpc from the solar circle (between $7 \mathrm{kpc}$ and $10.5 \mathrm{kpc}$ ), at different intervals of $|z|$. We find a positive slope that slightly decreases with $|z|$ from $d[\mathrm{Fe} / \mathrm{H}] / d R=0.0157 \pm 0.0002 \mathrm{dex} \mathrm{kpc}^{-1}$ for $|z|<0.5 \mathrm{kpc}$ to $0.0112 \pm 0.0007 \mathrm{dex} \mathrm{kpc}^{-1}$ in the range $2.5 \mathrm{kpc}<|z|<3.0 \mathrm{kpc}$ (the squares in Figure 4). We have measured $d[\mathrm{Fe} / \mathrm{H}] / d R$ by calculating the median of the chemical distribution in bins of $0.05 \mathrm{kpc}$ in $R$ and fitting a straight line to those medians, taking into account the $1 \sigma$ width of the distribution of the data in each 

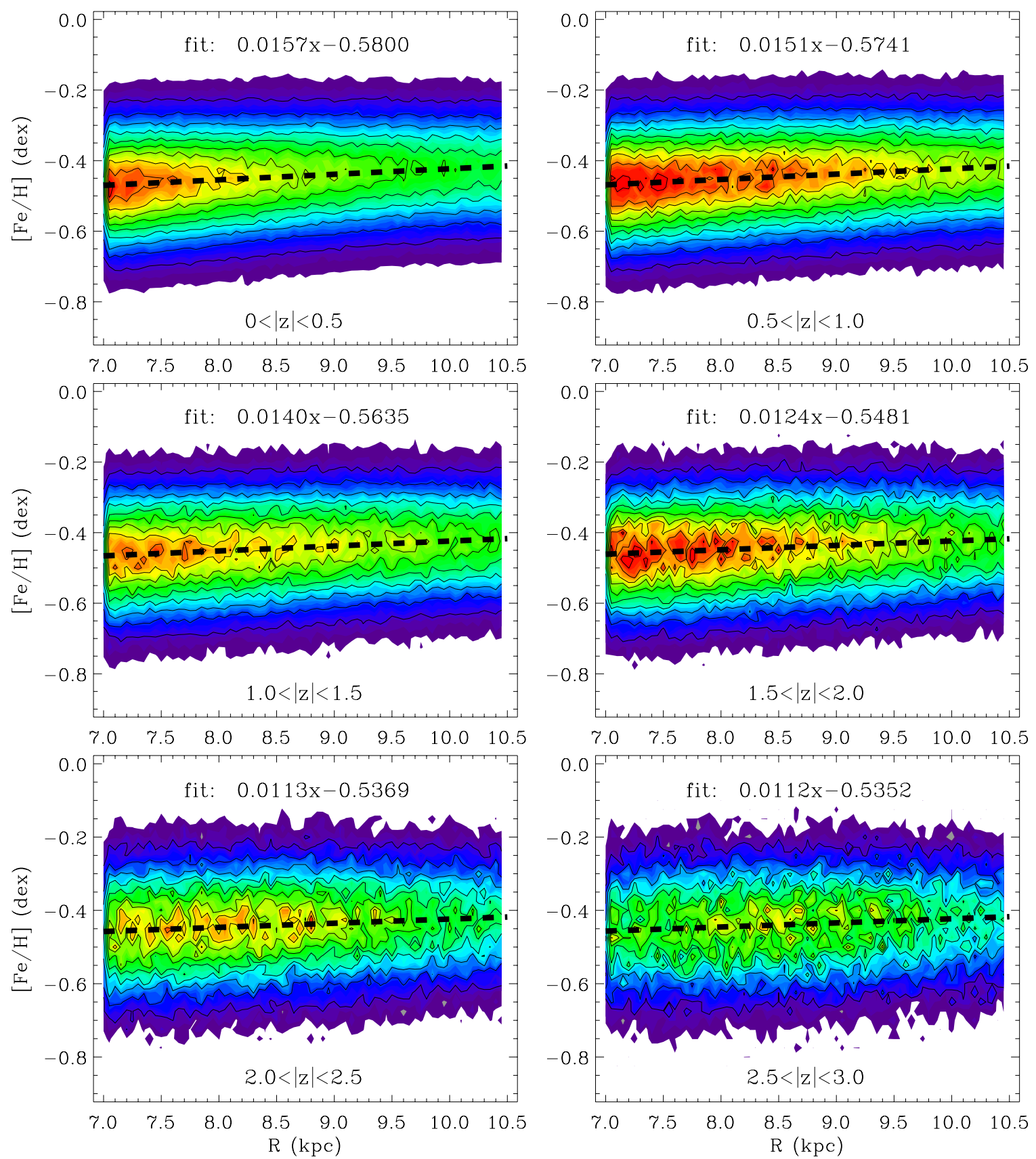

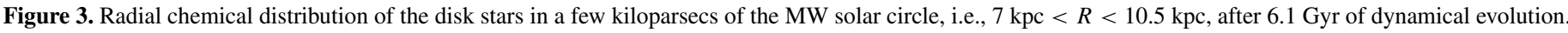
The different panels refers to different $|z|$ ranges. The black dashed lines are the best fit to the median values of [Fe/H] in each bin in $R$ (of $0.05 \mathrm{kpc}$ ).

(A color version of this figure is available in the online journal.)

bin; the gradient uncertainties are taken as the $1 \sigma$ error of the fit to the medians.

Recently, Carrell et al. (2012) examined the metallicity distribution (in $R$ and $|z|$ ) in the Galactic thick disk, using $\mathrm{F}, \mathrm{G}$ and K dwarf stars selected from Sloan Digital Sky Survey DR8. We compare our simulation with their sample of kinematically selected thick disk stars. Here we consider only the results derived by Carrell et al. (2012) for $|z|>1.5 \mathrm{kpc}$, in order to minimize the contamination of thin disk stars (Bensby et al. 2011).

Carrell et al. (2012) measured the metallicity gradient in the radial directions for stars in the annular region $(7.0 \mathrm{kpc}<R<$ $10.5 \mathrm{kpc}$ from the galactic center) and at several distances from the galactic plane. For thick disk stars they obtained an increas- ing $d[\mathrm{Fe} / \mathrm{H}] / d R$ with $|z|$, from $0.025 \pm 0.007 \mathrm{dex} \mathrm{kpc}^{-1}$ for $1.5 \mathrm{kpc}<|z|<2 \mathrm{kpc}$ to $0.041 \pm 0.016 \mathrm{dex} \mathrm{kpc}^{-1}$ for $2.5 \mathrm{kpc}<$ $|z|<3.0 \mathrm{kpc}$ (Isochrone Distance Method). These results are consistent, within $1 \sigma$ uncertainties, with a constant positive slope of $\approx 0.032 \mathrm{dex} \mathrm{kpc}^{-1}$ for the Isochrone Distance Method and a slope of $\approx 0.023 \mathrm{dex} \mathrm{kpc}^{-1}$ for the Photometric Distance Method.

A mild positive gradient of $0.010 \pm 0.005 \mathrm{dex} \mathrm{kpc}^{-1}$ was also measured by Hayden et al. (2013), among $\alpha$-enhanced stars with $T_{\text {eff }}>4200 \mathrm{~K}$ located between $|z|=1 \mathrm{kpc}$ and $|z|=2 \mathrm{kpc}$. Our results suggest a mildly decreasing positive slope of the order of $\sim 0.013 \mathrm{dex} \mathrm{kpc}-1$.

The evolution of the radial gradients in the solar neighborhood is illustrated in Figure 5. The initial radial gradients are given 


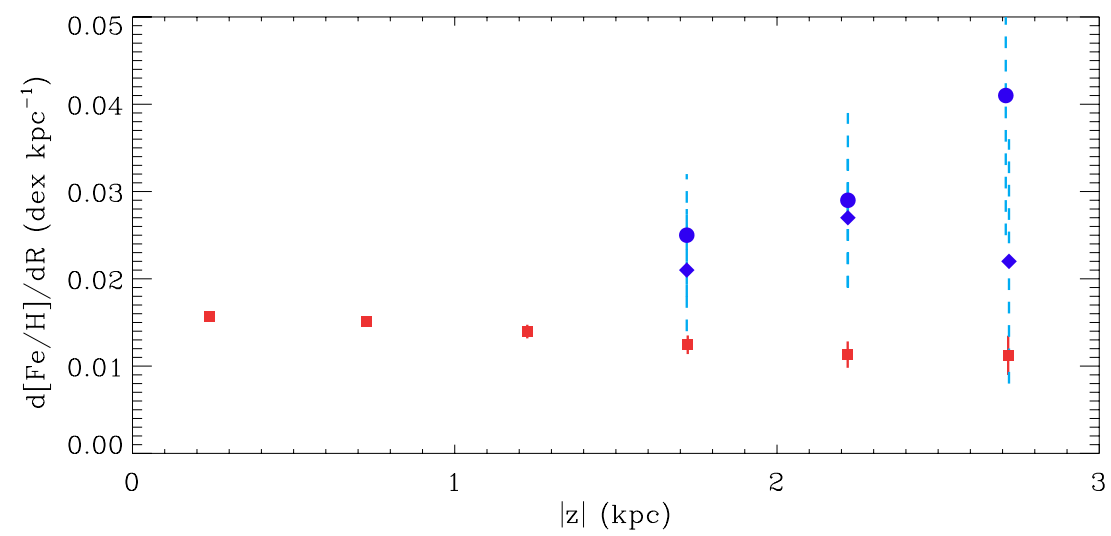

Figure 4. Radial metallicity gradients of the disk stars with $7 \mathrm{kpc}<R<10.5 \mathrm{kpc}$. The squares are our estimates of the metallicity gradients at $6.1 \mathrm{Gyr}$ from the dynamical $N$-body simulation. The abscissa of each point is the mean height of the particles in the respective bin (broad $0.5 \mathrm{kpc}$ in $R$ or $|z|$ ). The error bars on the vertical axis are $3 \sigma$ width of the distribution, as $1 \sigma$ errors would be hardly appreciated in this figure. Circles and diamonds are the gradients for $|z|>1.5 \mathrm{kpc}$ measured by Carrell et al. (2012) through the Isochrone Distance and the Photometric Distance, respectively.

(A color version of this figure is available in the online journal.)

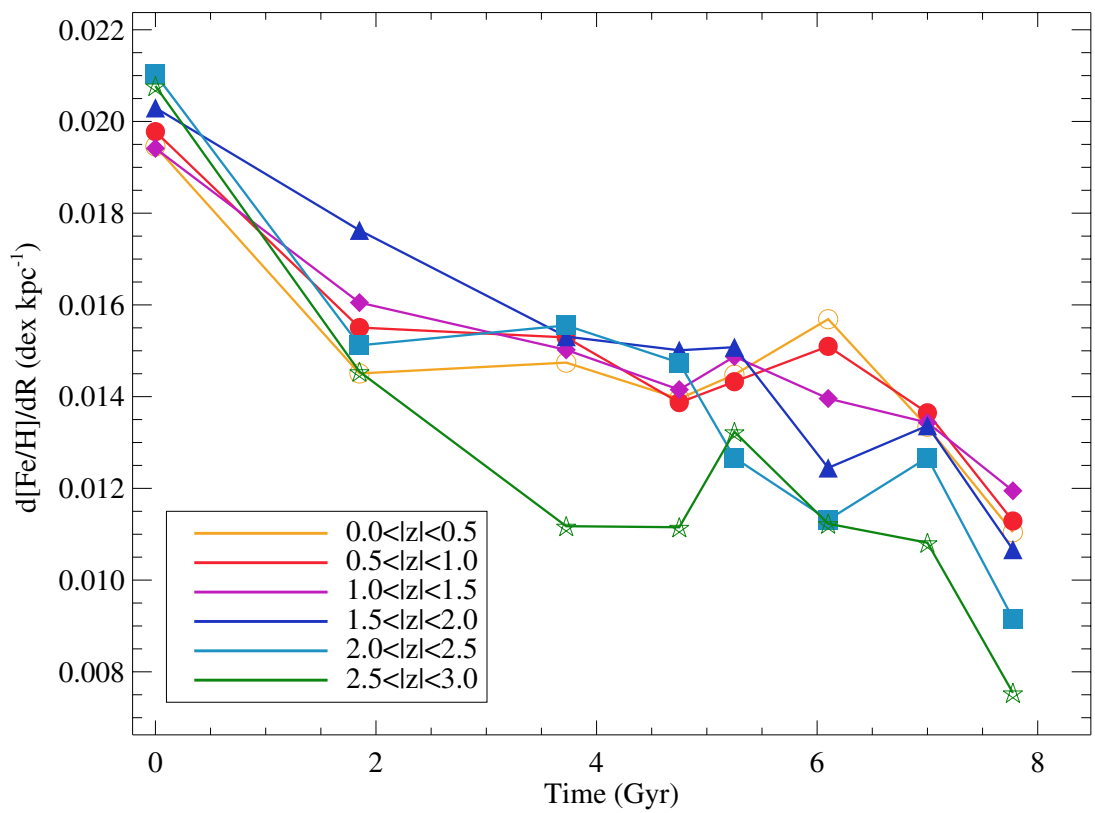

Figure 5. Evolution of the radial gradient in the solar annulus in several layers in $|z|$, namely $0 \mathrm{kpc}<|z|<0.5 \mathrm{kpc}$ (empty circles), $0.5 \mathrm{kpc}<|z|<1.0 \mathrm{kpc}$ (filled circles), $1.0 \mathrm{kpc}<|z|<1.5 \mathrm{kpc}$ (diamonds), $1.5 \mathrm{kpc}<|z|<2.0 \mathrm{kpc}$ (triangles), $2.0 \mathrm{kpc}<|z|<2.5 \mathrm{kpc}$ (squares), $2.5 \mathrm{kpc}<|z|<3.0 \mathrm{kpc}$ (stars).

(A color version of this figure is available in the online journal.)

by the model we use to assign the metallicities and, as there is no initial dependence on $|z|$, the radial gradients at different $|z|$ have approximately the same values. These profiles reach a substantial stability between $t \sim 1.8$ and $6 \mathrm{Gyr}$, for $0 \mathrm{kpc}<$ $|z|<2.5 \mathrm{kpc}$.

After 6 Gyr of evolution of our model galaxy, we do not see any obvious trend in the vertical metallicity gradients as a function of radial distance from the Galactic center. We find a significant, but tiny, positive $d[\mathrm{Fe} / \mathrm{H}] / d|z|$ of $0.0031 \pm 0.0003 \mathrm{dex} \mathrm{kpc}^{-1}$ for the stars in the radial and vertical ranges $7.0 \mathrm{kpc}<R<10.5 \mathrm{kpc}$ and $1 \mathrm{kpc}<|z|<3 \mathrm{kpc}$. On the other hand Carrell et al. (2012) measured $d[\mathrm{Fe} / \mathrm{H}] / d|z|=$ $-0.113 \pm 0.010 \mathrm{dex} \mathrm{kpc}^{-1}$ for the whole sample of thick disk stars in the same spatial region. Hayden et al. (2013) measured a value of $-0.22 \pm 0.02 \mathrm{dex} \mathrm{kpc}^{-1}$ for $d[\mathrm{M} / \mathrm{H}] / d|z|$ in the range $7 \mathrm{kpc}<R<9 \mathrm{kpc}$ and of $-0.13 \pm 0.04 \mathrm{dex} \mathrm{kpc}^{-1}$ in the range $9 \mathrm{kpc}<R<11 \mathrm{kpc}$.

\section{CONCLUSIONS}

The crucial role of a positive slope $d[\mathrm{Fe} / \mathrm{H}] / d R$ in the inner disk for the primordial chemical distribution to produce a positive rotation-metallicity correlation in the thick disk (Spagna et al. 2010; Lee et al. 2011) was already shown in Curir et al. (2012). In this work we have analyzed how the same cosmologically motivated chemical distribution allows us to constrain the influence of the dynamical evolution on the metallicity gradients observed today in the MW thick disk.

We have drawn the radial and vertical chemical distributions from the same simulated MW galaxy used in Curir et al. (2012), which includes a positive radial chemical gradient in the inner disk. At $6.1 \mathrm{Gyr}$, within the solar neighborhood $(7.0 \mathrm{kpc}<$ $R<10.5 \mathrm{kpc}$ ), we still obtain a positive slope for the radial chemical distribution in the inner region. This slope decreases in going from $|z|<0.5 \mathrm{kpc}$ to the region 
$2.5 \mathrm{kpc}<|z|<3 \mathrm{kpc}\left(d[\mathrm{Fe} / \mathrm{H}] / d R=0.016 \pm 0.002 \mathrm{dex} \mathrm{kpc}^{-1}\right.$ and $d[\mathrm{Fe} / \mathrm{H}] / d R=0.011 \pm 0.007 \mathrm{dex} \mathrm{\textrm {kc } ^ { - 1 }}$, respectively). The radial chemical gradients predicted by the model are at most within a factor of two of those observed by Carrell et al. (2012) with the Photometric Distance Method and by Hayden et al. (2013). Our final positive radial gradients are a direct consequence of our initial conditions, that include a positive radial gradient in the inner disk.

As shown in Figure 5, the radial gradients in the solar neighborhood are of the same order from $1.8 \mathrm{Gyr}$ to $6.1 \mathrm{Gyr}$, independently of the layer in $|z|$ (although some redistribution appears to take place). Such a stability seen between 2 and 6 Gyr is consistent with the stability of the rotation-metallicity correlation pointed out by Curir et al. (2012) in the same time range.

Furthermore, the vertical gradient in the final configuration of our simulation is very close to zero. This originates from the fact that we do not impose any dependence on $|z|$ in the initial chemical distribution. The lack of a $|z|$ dependence is also responsible from the lack of a trend for the radial gradients to increase with $|z|$.

We conclude that the secular disk evolution does not appear to be able to modify significantly the disk global chemical profiles, suggesting that the radial and vertical chemical distribution of the thick disk is likely to be a fossil signature of the original distribution.

Our results indicate that, if the positive radial metallicity gradient in the solar neighborhood will be confirmed by the observations of the MW thick disk, this is consistent with a thick disk population showing an early positive $[\mathrm{Fe} / \mathrm{H}]$ radial gradient in the inner disk $(R<10 \mathrm{kpc})$ and negative in the outer disk $(R>10 \mathrm{kpc})$. In the context of the inside-out formation scenarios of the galactic disk (Spitoni \& Matteucci 2011; Mott et al. 2013), such a gradient "inversion" derives from the strong infall of primordial gas that can occur at early times in the inner disk.

The authors thank R. Drimmel for accurate insightful comments.
We are grateful to F. Matteucci and E. Spitoni for providing us with the data of their chemical gradient model for a primordial disk. A.C., P.R.F., A.S. acknowledge the financial support of the PRIN-MIUR 2012, grant No 1.05.01.97.02, "Chemical and dynamical evolution of our Galaxy and of the galaxies of the Local Group."

A.L.S. and A.D. acknowledge partial support from the INFN grant InDark and from the grant Progetti di Ateneo/CSP TO Call2 20120011 of the Università di Torino.

M.G.L. and A.L.S. acknowledge partial support from MIUR and the Italian Space Agency through Contract No. I/058/10/0 (The Italian participation in the Gaia Mission).

\section{REFERENCES}

Allende Prieto, C., Beers, T. C., Wilhelm, R., et al. 2006, ApJ, 636, 804 Anders, F., Chiappini, C., Santiago, B. X., et al. 2013, arXiv:1311.4549 Bensby, T., Alves-Brito, A., Oey, M. S., Yong, D., \& Meléndez, J. 2011, ApJL, 735, L46

Boeche, C. the RAVE Collaboration 2013, arXiv:1309.6460

Brook, C. B., Kawata, D., Gibson, B. D., \& Freeman, K. C. 2004, ApJ, 612,894

Brook, C. B., Stinson, G. S., Gibson, B. K., et al. 2012, MNRAS, 426, 690

Brunetti, M., Chiappini, C., \& Pfenninger, D. 2011, A\&A, 534, A75

Carrell, K., Chen, Y., \& Zhao, G. 2012, AJ, 144, 185

Cheng, J. Y., Rockosi, C. M., Morrison, H. L., et al. 2012, ApJ, 746, 149

Cresci, G., Mannucci, F., Maiolino, R., et al. 2010, Natur, 467, 811

Curir, A., Lattanzi, M. G., Spagna, A., et al. 2012, A\&A, 545, A133

Hayden, M. R., Holtzman, J. A., Bovy, J., et al. 2013, arXiv:1311.4569

Kubryk, M., Prantzos, N., \& Athanassoula, E. 2013, MNRAS, 436, 1479

Lee, Y. S., Beers, T. C., An, D., et al. 2011, ApJ, 738, 187

Loebman, S., Roškar, R., Debattista, V. P., et al. 2011, ApJ, 737, 8

Marsakov, V. A., \& Borkova, T. V. 2005, AstL, 31, 515

Minchev, I., Famaey, B., Quillen, A. C., et al. 2012, A\&A, 548, A126

Mott, A., Spitoni, E., \& Matteucci, F. 2013, MNRAS, 435, 2918

Navarro, J. F., Frenk, C. S., \& White, S. D. M. 1997, ApJ, 190, 493

Roškar, R., Debattista, V. P., \& Loebman, S. R. 2013, MNRAS, 433, 976

Roškar, R., Debattista, V. P., Stinson, G. S., et al. 2008, ApJL, 675, L65

Spagna, A., Lattanzi, M. G., Re Fiorentin, P., \& Smart, R. L. 2010, A\&A, 510, L4

Spitoni, E., \& Matteucci, F. 2011, A\&A, 531, 72

Springel, V. 2005, MNRAS, 364, 1105 